\title{
Inequalities for Covering Codes
}

\author{
A. R. CALDERBANK AND N. J. A. SLOANE, FELlow, IEEE
}

\begin{abstract}
Any code $C$ with covering radius $R$ must satisfy a set of linear inequalities that involve the Lloyd polynomial $L_{R}(x)$; these generalize the sphere bound. The "syndrome graphs" associated with a linear code $C$ help to keep track of low weight vectors in the same coset of $C$ (if there are too many such vectors $C$ cannot exist). As illustrations it is shown that $t[17,10]=3$ and $t[23,15]=3$, where $t[n, k]$ is the smallest covering radius of any $[n, k]$ code.
\end{abstract}

\section{INTRODUCTION}

$\mathrm{T}$ HE Delsarte-MacWilliams inequalities have enabled linear programming to be applied successfully to error-correcting codes (see for example [4]). The inequalities presented here (in Theorems 1,2 and Corollaries 3, 4) are a partial analog for covering codes. Although several other recent papers have given bounds for covering codes [1], [5], [7], the results given here appear to be new. The technique of obtaining bounds by counting syndromes (used in the second proof of Theorem 2) was also used by Brualdi et al. [1]. The underlying principle is the fact that if $C$ is a linear $[n, k]$ code with covering radius $R$, then every $(n-k)$-tuple can be written as a sum of at most $R$ columns of $H[2$, sec. I-A]. The bookkeeping involved in checking this condition is facilitated by the "syndrome graphs" introduced in Section III. In Section IV we show $t[17,10]=3$, and the final section contains a list of the known improvements to the table of $t[n, k]$ given in [3]. (Recall that $t[n, k]$ is the minimal covering radius of any binary code of length $n$ and dimension $k$.)

We restrict ourselves to binary codes; there is a straightforward generalization to other fields. Our notation throughout is that a linear code $C$ has weight distribution $\left\{A_{i}\right\}$ and the dual $C^{\perp}$ has weight distribution $\left\{B_{i}\right\}$.

\section{Linear INequalities For COVERING CoDes}

Suppose $C$ is a (not necessarily linear) binary code of length $n$ and covering radius $R$. As in [4, ch. 5], we represent vectors $u=\left(u_{1}, \cdots, u_{n}\right) \in F_{2}^{n}$ by their images $z^{u}=z_{1}^{u_{1}} \cdots z_{n}^{u_{n}}$ in the group algebra $\boldsymbol{Q F _ { 2 } ^ { n }}$, and define

$$
\begin{aligned}
& \chi_{v}\left(z^{u}\right)=(-1)^{u \cdot v}, \\
& \chi_{v}(C)=\sum_{c \in C} \chi_{v}\left(z^{c}\right) .
\end{aligned}
$$

Manuscript received November 23, 1987; revised February 26, 1988. The authors are with the Mathematical Sciences Research Center, AT\&T Bell Laboratories, Murray Hill, NJ 07974.

IEEE Log Number 8824280.
Let

$$
\begin{aligned}
K_{j}(x ; n) & =\sum_{i=0}^{j}(-1)^{i}\left(\begin{array}{c}
x \\
i
\end{array}\right)\left(\begin{array}{c}
n-x \\
j-i
\end{array}\right), \\
L_{j}(x) & =\sum_{i=0}^{j} K_{i}(x ; n)=K_{j}(x-1 ; n-1),
\end{aligned}
$$

$j=0,1, \cdots, n$, be Krawtchouk and Lloyd polynomials, respectively [4].

Theorem 1: For any subspace $U \subseteq F_{2}^{n}$ and any vector $v \in \boldsymbol{F}_{2}^{n}$ we have

$$
\sum_{u \in U} \chi_{v}\left(z^{u}\right) \chi_{u}(C) L_{R}(\mathrm{wt}(u)) \geq 2^{n}
$$

Proof: Since $C$ has covering radius $R$, in the group algebra we have

$$
\left(\sum_{c \in C} z^{c}\right)\left(\sum_{i=0}^{R} Y_{i}\right)=\sum_{w \in F_{2}^{n}} \alpha_{w} z^{w},
$$

where

$$
Y_{i}=\sum_{w t(w)=i} z^{w}
$$

and the coefficients $\alpha_{w}$ are positive integers. We apply $\chi_{u}$ to both sides of (4) and use

$$
\chi_{u}\left(Y_{i}\right)=\sum_{\mathrm{wt}(w)=i}(-1)^{u \cdot w}=K_{i}(\mathrm{wt}(u) ; n)
$$

$[4$, p. 135$]$ and (2) to obtain

$$
\chi_{u}(C) L_{R}(w t(u))=\sum_{w \in F_{2}^{n}} \alpha_{w}(-1)^{u \cdot w}
$$

By multiplying both sides by $(-1)^{u \cdot v}$ and summing on $u \in U$, we have

$$
\begin{aligned}
& \sum_{u \in U} \chi_{v}\left(z^{u}\right) \chi_{u}(C) L_{R}(\mathrm{wt}(u)) \\
&=\sum_{w \in F_{2}^{n}} \alpha_{w} \sum_{u \in U}(-1)^{u \cdot(v+w)} .
\end{aligned}
$$

But

$$
\sum_{u \in U}(-1)^{u \cdot x}= \begin{cases}|U|, & \text { if } x \in U^{\perp} \\ 0, & \text { otherwise, }\end{cases}
$$

so the right side of $(6)$ is at least

$$
|U| \sum_{\substack{w \in F_{2}^{n} \\ w+v \in U^{\perp}}} \alpha_{w} \geq|U|\left|U^{\perp}\right|=2^{n},
$$

which is the desired result. 
From now on we restrict our attention to linear codes.

Theorem 2: Let $C$ be a linear $[n, k] R$ code, i.e. one with covering radius $R$. Choose any $m$ linearly independent vectors $u_{1}, \cdots, u_{m}$ in $C^{\perp}(1 \leq m \leq n-k)$, and $m$ arbitrary signs $\epsilon_{1}, \cdots, \epsilon_{m}$ (equal to \pm 1 ). If $u \in C^{\perp}$ is a linear combination of the $u_{i}$, say

$$
u=u_{i_{1}}+\cdots+u_{i_{\alpha}},
$$

set $\epsilon(u)=\epsilon\left(i_{1}\right) \cdots \epsilon\left(i_{\alpha}\right)$; otherwise set $\epsilon(u)=0$. Then

$$
\sum_{u \in C^{\perp}} \epsilon(u) L_{R}(\mathrm{wt}(u)) \geq 2^{n-k} .
$$

\section{Remarks:}

i) Since $L_{R}(0)=\sum_{i=0}^{R}\left(\begin{array}{c}n \\ i\end{array}\right)$, we can write (8) as

$$
\sum_{i=0}^{R}\left(\begin{array}{c}
n \\
i
\end{array}\right)-2^{n-k}+\sum_{\substack{u \in C^{\perp} \\
u \neq 0}} \epsilon(u) L_{R}(\mathrm{wt}(u)) \geq 0 .
$$

The sphere bound

$$
\sum_{i=0}^{R}\left(\begin{array}{c}
n \\
i
\end{array}\right)-2^{n-k} \geq 0
$$

is the case $m=0$.

ii) Theorem 2 can be stated more succinctly as follows: if $U$ is a subspace of $C^{\perp}$ and $\epsilon$ is any map from $C^{\perp}$ to $\{-1,0,+1\}$ satisfying $\epsilon(u)=0$ for $u \in C^{\perp} \backslash U$ and $\epsilon(u+$ $v)=\epsilon(u) \epsilon(v)$ for $u, v \in U$, then (8) holds.

iii) Although Theorem 2 follows from Theorem 1, we also give a direct proof involving the choice of a parity check matrix, since this approach motivates the rest of the paper.

First proof of Theorem 2: In Theorem 1 let $C$ be linear, $U \subseteq C^{\perp}$, and choose $v$ so that $\chi_{v}\left(z^{u}\right)=\epsilon(u)$ holds for all $u \in U$. Then (8) follows from (3) and (7).

Second proof: Let $u_{1}, \cdots, u_{m}$ be the first $m$ rows of a parity check matrix $H$ for $C$. Define $\pi_{i}=0$ or 1 by $\epsilon_{i}=(-1)^{\pi_{i}}(i=1, \cdots, m)$. Then for any $a \in F_{2}^{n}$,

$$
\prod_{i=1}^{m} \frac{1+\epsilon_{i}(-1)^{a \cdot u_{i}}}{2}
$$

is equal to 1 if $a \cdot u_{i}=\pi_{i}$ for all $i=1, \cdots, m$, and is otherwise 0 . Therefore

$$
\sum_{\operatorname{wt}(a) \leq R} \prod_{i=1}^{m} \frac{1+\epsilon_{i}(-1)^{a \cdot u_{i}}}{2} \geq 2^{n-k-m},
$$

since the left side is the number of linear combinations of at most $R$ columns of $H$ whose sum begins $\pi_{1} \pi_{2} \cdots \pi_{m}$, and this must be at least as large as the number of $(n-k)$-tuples with the same beginning. Equation (8) follows by expanding (11), and applying (5) and (2).

Corollary 3: If $w$ is any nonzero weight in $C^{\perp}$ then

a)

$$
\sum_{i=0}^{R}\left(\begin{array}{c}
n \\
i
\end{array}\right)-2^{n-k} \geq\left|L_{R}(w)\right|
$$

and

$$
M(w) \geq 0,
$$

where

$$
M(x)=L_{R}(0)-2^{n-k}-L_{R}(x) .
$$

Proof: a) is the case $m=1$ of Theorem 2 ; b) is an immediate consequence.

Corollary 4: If $u_{1}, u_{2}, u_{3}=u_{1}+u_{2}$ are nonzero vectors in $C^{\perp}$ of weights $w_{1}, w_{2}, w_{3}$ respectively, then

$$
\sum_{i=0}^{R}\left(\begin{array}{l}
n \\
i
\end{array}\right)-2^{n-k}+\epsilon_{1} L_{R}\left(w_{1}\right)+\epsilon_{2} L_{R}\left(w_{2}\right)+\epsilon_{1} \epsilon_{2} L_{R}\left(w_{3}\right) \geq 0
$$

holds for all choices of signs $\epsilon_{1}= \pm 1, \epsilon_{2}= \pm 1$; and

$$
\text { b) } \quad M\left(w_{1}\right)+M\left(w_{2}\right)-M\left(w_{3}\right) \geq 0 \text {. }
$$

Proof: a) is the case $m=2$ of Theorem 2; b) follows by choosing $\epsilon_{1}=\epsilon_{2}=-1$.

\section{Remarks:}

i) Equation (16) states that the three numbers $M\left(w_{1}\right)$, $M\left(w_{2}\right), M\left(w_{3}\right)$ are a possible set of edge lengths of a triangle (which may have zero area-for example if $M\left(w_{1}\right)$ $\left.+M\left(w_{2}\right)=M\left(w_{3}\right)\right)$.

ii) Although assertions (13) and (16) are generally the most useful, there are many applications of the more general results (8), (12), (15).

iii) Lloyd's theorem for linear codes. Let $C$ be a perfect $[n, k] R$ code. Consider the graph $\Gamma$ with vertices the cosets of $C$ and where two cosets $x, y$ are joined if and only if wt $(x+y)=1$. If we choose a parity check matrix $H$, then we may label the vertices of $\Gamma$ with syndromes with respect to $H$. Vertices labeled $s, t$ are joined in $\Gamma$ if and only if $s+t$ is a column of $H$. The graph $\Gamma$ is regular with valency $n$ and has eigenvalues $n-2 w_{j}$ with multiplicity $B_{w_{j}}$, where $B_{w_{j}}$ is the number of codewords in $C^{\perp}$ with weight $w_{j}$. Since the diameter of $\Gamma$ is $R$, there are at least $R$ nonzero weights in $C^{\perp}$. Since $C$ is perfect we have

$$
L_{R}(0)=2^{n-k},
$$

and for every nonzero weight $w$ in $C^{\perp}$ we have

$$
M(w)=L_{R}(0)-2^{n-k}-L_{R}(w)=0 .
$$

We conclude that the polynomial $L_{R}(x)$ of degree $R$ has exactly $R$ distinct integer roots in $[0, n]$.

Corollary 5: Suppose there is an integer $\beta(0 \leq \beta \leq n)$ such that for all integers $w(0 \leq w \leq n)$ either

$$
M(w) \leq \beta \quad \text { or } \quad M(w) \geq 2 \beta+1 .
$$

Then

$$
\left\{u \in C^{\perp}: M(w \mathrm{t}(u)) \leq \beta\right\}
$$

together with the zero vector is a linear subcode of $C^{\perp}$.

Proof: Let $u_{1}, u_{2} \in C^{\perp}$ be linearly independent vectors with $M\left(\mathrm{wt}\left(u_{i}\right)\right) \leq \beta(i=1,2)$. We must show $M\left(\mathrm{wt}\left(u_{1}\right.\right.$ $\left.\left.+u_{2}\right)\right) \leq \beta$. Let $w_{i}=\operatorname{wt}\left(u_{i}\right), \quad w_{3}=\mathrm{wt}\left(u_{1}+u_{2}\right)$. Then 
$M\left(w_{1}\right)+M\left(w_{2}\right) \geq M\left(w_{3}\right) \geq 0$, so $M\left(w_{3}\right) \leq 2 \beta$. Therefore $M\left(w_{3}\right) \leq \beta$, by hypothesis.

Corollary 6: The zero vector and the vectors $u \in C^{\perp}$ with $M(w t(u))=0$ form a linear subcode of $C^{\perp}$.

Proof: This is the case $\beta=0$.

Examples:

a) It was shown in [3] that $t[23,15]=2$ or 3 . Suppose $C$ is a $[23,15] 2$ code. The corresponding values of $L_{2}(x)$ and $M(x)$ for $0 \leq x \leq 23$ are as follows:

\begin{tabular}{rrrrrrrrr}
\hline$x:$ & 0 & 1 & 2 & 3 & 4 & 5 & 6 & 7 \\
$L_{2}(x):$ & 277 & 231 & 189 & 151 & 117 & 87 & 61 & 39 \\
$M(x):$ & -256 & -210 & -168 & -130 & -96 & -66 & -40 & -18 \\
$x:$ & 8 & 9 & 10 & 11 & 12 & 13 & 14 & 15 \\
$L_{2}(x):$ & 21 & 7 & -3 & -9 & -11 & -9 & -3 & 7 \\
$M(x):$ & 0 & 14 & 24 & 30 & 32 & 30 & 24 & 14 \\
$x:$ & 16 & 17 & 18 & 19 & 20 & 21 & 22 & 23 \\
$L_{2}(x):$ & 21 & 39 & 61 & 87 & 117 & 151 & 189 & 231 \\
$M(x):$ & 0 & -18 & -40 & -66 & -96 & -130 & -168 & -210 \\
\hline
\end{tabular}

From (13), the nonzero codewords in $C^{\perp}$ have weights in the range $\{8,9, \cdots, 16\}$, and from Corollary 6 the codewords of weights $0,8,16$ form a linear subcode.

We shall continue this example in Section III, and show that $C$ does not exist.

b) From [3], $t[16,7]=3$ or 4 . Suppose $C$ is a $[16,7] 3$ code. The corresponding values of $L_{3}(x)$ and $M(x)$ are as follows:

\begin{tabular}{rrrrrrrrrr}
\hline$x:$ & 0 & 1 & 2 & 3 & 4 & 5 & 6 & 7 & 8 \\
$L_{3}(x):$ & 697 & 455 & 273 & 143 & 57 & 7 & -15 & -17 & -7 \\
$M(x):$ & -512 & -270 & -88 & 42 & 128 & 178 & 200 & 202 & 192 \\
$x:$ & 9 & 10 & 11 & 12 & 13 & 14 & 15 & 16 & \\
$L_{3}(x):$ & 7 & 17 & 15 & -7 & -57 & -143 & -273 & -455 & \\
$M(x):$ & 178 & 168 & 170 & 192 & 242 & 328 & 458 & 640 & \\
\hline
\end{tabular}

From (13), the nonzero codewords in $C^{\perp}$ have weights $w \geq 3$. In this case (12) yields the additional result that $w \leq 14$.

At present it is not known if $C$ exists.

\section{SYNDROME GRAPHS}

Every nonzero codeword $c \in C$ specifies a dependence among the columns of $H$. In particular, the existence of a codeword in $C$ of weight $w \leq 2 R$ implies that two sets of at most $R$ columns of $H$ have equal sums (usually in several ways). For example, if $c=111100 \cdots 0 \in C$, we have the identities

$$
\begin{aligned}
& h_{1}+h_{2}=h_{3}+h_{4}, \\
& h_{1}+h_{3}=h_{2}+h_{4}, \\
& h_{1}+h_{4}=h_{2}+h_{3},
\end{aligned}
$$

where $h_{i}$ is the $i$ th column of $H$. Each such identity reduces the number of distinct sums of at most $R$ columns of $H$. If there are enough identities it may be possible to show that the code cannot exist. (Such identities indicate that there are low weight vectors in the same coset of $C$.)

We now define certain "syndrome graphs" $G_{\pi_{1} \cdots \pi_{m}}$, which make it possible to record and utilize even partial information of this type.
As in Section II, let $u_{1}, \cdots, u_{m} \in C^{\perp}$ be the first $m$ rows of a parity check matrix $H(1 \leq m \leq n-k)$. For each binary vector $\pi_{1} \cdots \pi_{m}$ we define a graph $G_{\pi_{1} \cdots \pi_{m}}$ as follows. The vertices are the subsets $\left\{i_{1}, \cdots, i_{r}\right\} \subseteq\{1, \cdots, n\}$ $(0 \leq r \leq R)$ for which the binary vector $a$ supported on that subset satisfies $a \cdot u_{j}=\pi_{j}$ for all $j=1, \cdots, m$. Two vertices $\left\{i_{1}, \cdots, i_{r}\right\},\left\{j_{1}, \cdots, j_{s}\right\}$ are joined by an edge if and only if

$$
\sum_{\rho=1}^{r} h_{i_{\rho}}=\sum_{\sigma=1}^{s} h_{j_{\sigma}} .
$$

Proposition 7: The syndrome graph $G_{\pi_{1} \ldots \pi_{m}}$ contains exactly $2^{n-k-m}$ connected components, each of which is a complete graph.

Proof: It follows from the definition that the number of components in this graph is equal to the number of distinct sums $f=\left(f_{1}, \cdots, f_{n-k}\right)^{t r}$ of at most $R$ columns of $H$ for which $f_{1}=\pi_{1}, \cdots, f_{m}=\pi_{m}$. Since $C$ has covering radius $R$, this number is exactly $2^{n-k-m}$. It also follows from the definition that if node $V_{1}$ is joined to node $V_{2}$, and $V_{2}$ to $V_{3}$, then $V_{1}$ is joined to $V_{3}$; thus every component is a complete subgraph.

The merit of this graphical approach is that each time one can deduce-for example from the DelsarteMacWilliams identities - that a codeword of weight at most $2 R$ exists in $C$, the number of known edges in one or more of the graphs $G_{\pi_{1} \cdots \pi_{m}}$ increases. And of course a graph with many edges cannot have too many connected components.

This leads to the following proposition.

Proposition 8: Suppose the $i$ th connected component of the syndrome graph $G_{\pi_{1} \cdots \pi_{m}}$ contains $v_{i}$ vertices and $e_{i}=$ $v_{i}\left(v_{i}-1\right) / 2$ edges $\left(1 \leq i \leq K=2^{n-k-m}\right)$, and let $v=\sum v_{i}$, $e=\sum e_{i}$ be the total number of vertices and edges, respectively. Then the nonnegative integers $\delta_{i}=v_{i}-1(1 \leq i \leq K)$ satisfy

$$
\begin{aligned}
& \sum_{i=1}^{K} \delta_{i}=v-2^{n-k-m}, \\
& \sum_{i=1}^{K} \delta_{i}^{2}=2 e-v+2^{n-k-m} .
\end{aligned}
$$

Proof: This follows immediately from the definition of $\delta_{i}$.

Remarks:

i) If it is known only that the syndrome graph $G_{\pi_{1}, \cdots, \pi_{m}}$ contains at least $\eta$ edges, then Proposition 8 implies that there exist nonnegative integers $\left\{\delta_{i}\right\}$ satisfying

$$
\begin{aligned}
& \sum_{i=1}^{K} \delta_{i}=v-2^{n-k-m}, \\
& \sum_{i=1}^{K} \delta_{i}^{2} \geq 2 \eta-v+2^{n-k-m} .
\end{aligned}
$$


ii) It is worth emphasizing that $G_{\pi_{1} \ldots \pi}$ depends both on the choice of $u_{1}, \cdots, u_{m} \in C^{\perp}$ and on the binary vector $\pi_{1} \cdots \pi_{m}$

iii) It is sometimes useful to consider how the different syndrome graphs are related. There is a "parent" graph $\mathscr{G}$ with $L_{R}(0)$ vertices labeled by all subsets $\left\{i_{1}, \cdots, i_{r}\right\} \subseteq$ $\{1, \cdots, n\}, 0 \leq r \leq R$, with edges defined by (17). Each $G_{\pi_{1} \cdots \pi_{m}}$ is an induced subgraph of $\mathscr{G}$. Furthermore if $u_{i_{1}}, \cdots, u_{i_{s}}$ is a subset of $u_{1}, \cdots, u_{m}$, then $G_{\pi_{1} \cdots \pi_{m}}$ is an induced subgraph of $G_{\pi_{i_{1}} \cdots \pi_{i_{i}}}$.

iv) Consider the case $m \stackrel{\pi_{i_{s}}}{=} 1, \pi_{1}=1$. Then $M\left(\right.$ wt $\left.\left(u_{1}\right)\right)=0$ if and only if $G_{1}$ contains no edges (for then equality must hold in (11)). More generally, if $u_{1}, \cdots, u_{m}$ are such that $M\left(w t\left(u_{i}\right)\right)=0(1 \leq i \leq m)$, then all the syndrome graphs $G_{\pi_{1}} \cdots \pi_{m}$ for which at least one $\pi_{i}$ is 1 contain no edges (this follows from the last sentence of the previous remark).

Example a) (Continued):

Let $C$ be a $[23,15] 2$ code, and let $\left\{A_{i}\right\},\left\{B_{i}\right\}$ be the weight distributions of $C, C^{\perp}$ respectively. From Section II and the tables in [3], [6] we have $A_{1}=A_{2}=0, B_{1}=\cdots$ $=B_{7}=B_{17}=\cdots=B_{23}=0, B_{8} \geq 1$. We now use linear programming to minimize $A_{3}+A_{4}$ subject to the DelsarteMacWilliams constraints; the result is $A_{3}+A_{4} \geq 12.33 \cdots$, i.e. $A_{3}+A_{4} \geq 13$.

Suppose $C^{\perp}$ contains a codeword $u_{1}$ of weight 12 . The corresponding syndrome graph $G_{0}$ (i.e., taking $m=1, \pi_{1}=$ 0 ) has $v=1+\left(\begin{array}{r}12 \\ 2\end{array}\right)+11+\left(\begin{array}{r}11 \\ 2\end{array}\right)=133$ vertices. Furthermore $G_{0}$ contains at least one edge for every codeword of weight 3 or 4 in $C$, i.e. $e \geq 13$. (For example, suppose $u_{1}=$ $11 \cdots 100 \cdots 0 \in C^{\perp}$, of weight 12 . If $c=110 \cdots 010 \cdots 0$ $\in C$, of weight 3 , then there is an edge in $G_{0}$ joining vertex $\{1,2\}$ to vertex $\{13\}$. On the other hand if $c=$ $0 \cdots 01110 \cdots 0 \in C$, the three vertices $\{13,14\}$, $\{13,15\},\{14,15\}$ in $G_{0}$ are joined by three edges to form a triangle (similarly if $c$ has weight 4 ). The edges determine $c$, and so distinct $c$ 's produce distinct edges.)

From (20) and (21) it follows that $\sum_{i=1}^{128} \delta_{i}=5$ and $\sum_{i=1}^{128} \delta_{i}^{2}$ $\geq 21$, which implies $\delta_{j}=5$ for some $j$ and $\delta_{i}=0$ for $i \neq j$. We take $j=1$. Thus $G_{0}$ contains one component with six vertices (and at least 13 edges) and 127 isolated vertices. It is now easy to check that there is no way to choose 13 vectors of weight 3 or 4 orthogonal to $u_{1}$ for which the corresponding edges are restricted to only six vertices. Thus $C^{\perp}$ contains no vector of weight 12 , i.e. $B_{12}=0$. Similar arguments apply if $u_{1}$ has weight 11 or 13 , and we obtain $B_{11}=B_{12}=B_{13}=0$. Linear programming now shows that there is no feasible solution to the Delsarte-MacWilliams constraints. Thus $C$ does not exist, and we have established the following result.

Theorem 9:

$$
t[23,15]=3 \text {. }
$$

Remark: We originally proved Theorem 9 without using a computer. To save space we omit this argument.

\footnotetext{
${ }^{1}$ A graph $H$ is an induced subgraph of $G$ if the vertices of $H$ are a subset of the vertices of $G$, and the edges of $H$ are just the edges of $G$ that join these vertices.
}

$$
\text { IV. } t[17,10]=3
$$

Theorem 10:

$$
t[17,10]=3 .
$$

Proof: From [3], $t[17,10]=2$ or 3. Suppose $C$ is a $[17,10] 2$ code. The corresponding values of $L_{2}(x)$ and $M(x)$ are as follows:

\begin{tabular}{rrrrrrrrrr}
\hline$x:$ & 0 & 1 & 2 & 3 & 4 & 5 & 6 & 7 & 8 \\
$L_{2}(x):$ & 154 & 120 & 90 & 64 & 42 & 24 & 10 & 0 & -6 \\
$M(x):$ & -128 & -94 & -64 & -38 & -16 & 2 & 16 & 26 & 32 \\
$x:$ & 9 & 10 & 11 & 12 & 13 & 14 & 15 & 16 & 17 \\
$L_{2}(x):$ & -8 & -6 & 0 & 10 & 24 & 42 & 64 & 90 & 120 \\
$M(x):$ & 34 & 32 & 26 & 16 & 2 & -16 & -38 & -64 & -94 \\
\hline
\end{tabular}

From (13), the nonzero weights in $C^{\perp}$ are in the range $5 \leq w \leq 13$; and from [6], $C^{\perp}$ has $d_{\min }=5$ or 6 . From (16), there is at most one vector of weight 5 in $C^{\perp}$, and also if there is such a vector, then there can be no vector of weight 6 . Linear programming now shows there is no feasible solution to the Delsarte-MacWilliams inequalities if $B_{5}=1, B_{6}=0$. We conclude that $C^{\perp}$ has $d_{\min }=6$. Equation (16) also shows that any vector disjoint from a vector of weight 6 in $C^{\perp}$ must also have weight 6 (and by a dimension argument there must be such a vector).

Let $u_{1}, u_{2} \in C^{\perp}$ be disjoint vectors of weight 6 , say $u_{1}=11111100 \cdots 0, u_{2}=00000011111100000$. Let $D$ be the subcode of $C^{\perp}$ that vanishes on the last five coordinates, and let $D^{\prime}$ be the projection of $C^{\perp}$ onto the last five coordinates, so that $\operatorname{dim} D+\operatorname{dim} D^{\prime}=\operatorname{dim} C^{\perp}=7$, and $\operatorname{dim} D \geq 2$ (since $u_{1}, u_{2} \in D$ ).

Case i): $\operatorname{Dim} D=2$. Then $\operatorname{dim} D^{\prime}=5, D^{\prime}=F_{2}^{5}$, so in particular $C^{\perp}$ contains vectors $u_{3}, \cdots, u_{7}$ whose last five coordinates are $10000, \cdots, 00001$ respectively. A computer program considered all ways to choose $u_{3}$ and $u_{4} \in C^{\perp}$, ending with 10000 and 01000 respectively, and such that $\left\{u_{1}, u_{2}, u_{3}, u_{4}\right\}$ satisfy (8) for all choices of signs. There is an essentially unique solution (after taking account of obvious symmetries), which is shown in Fig. 1. The same program then found that there is no way to choose the fifth row $u_{5}$ to end with 00100 and such that $\left\{u_{1}, \cdots, u_{5}\right\}$ satisfy (8) for all choices of signs.

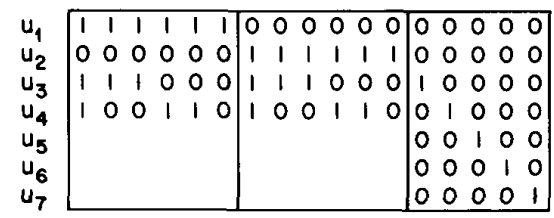

Fig. 1. Partial parity check matrix for $[17,10] 2$ code

Case ii): $\operatorname{Dim} D \geq 3$. The computer program found all ways to adjoin two further vectors $u_{3} \in D, u_{4} \in C^{\perp}$ to $u_{1}, u_{2}$ so that $\left\{u_{1}, u_{2}, u_{3}, u_{4}\right\}$ satisfy (8) for all choices of signs. There are 27 essentially distinct solutions $\left\{u_{3}, u_{4}\right\}$ (after allowing for obvious symmetries), but in each solution $u_{3}$ has weight six and intersects both $u_{1}$ and $u_{2}$ in 
three coordinates. We may therefore take $u_{3}$ as follows:

$$
\begin{array}{rrrrrr}
u_{1}= & 111 & 111 & 000 & 000 & 00000 \\
u_{2}= & 000 & 000 & 111 & 111 & 00000 \\
u_{3}= & 111 & 000 & 111 & 000 & 00000 \\
u_{4}= & \alpha & \beta & \gamma & \delta & \epsilon
\end{array}
$$

Furthermore, in each solution the last five coordinates of $u_{4}$ (denoted by $\epsilon$ in (22)) have weight at least 2 . This shows that $\operatorname{dim} D$ is exactly $3, \operatorname{dim} D^{\prime}=4$, and $D^{\prime}$ is the $[5,4]$ even weight code. Therefore $\epsilon$ has even weight. This reduces the number of inequivalent choices for $u_{4}$ (and similarly for the remaining three rows of $H$ ) to 12 , and we observe that none of these vanishes on any of the first four blocks of three coordinates (i.e., none of $\alpha, \beta, \gamma, \delta$ ever vanishes in (22)). But this is impossible, since there are four rows of $H$ to be completed, and they cannot all be distinct on the first three coordinates. Thus $C$ does not exist.

\section{IMPROVEMENTS TO THE TABLE OF $t[n, k]$}

The known improvements to the table of $t[n, k]$ given in [3] are as follows: From [5]: $t[15,6]=4$. From [7]: $t[23,6]$ $=7, \quad t[25,9]=6, \quad t[26,12]=5, \quad t[28,6] \geq 9, \quad t[29,8] \geq 8$, $t[30,7] \geq 9, t[32,10] \geq 8, t[33,6] \geq 11$. From $[1]: t[27,19]=$ $2, t[41,29]=3, t[42,33]=2, t[58,48]=2$ (these four values are attained by normal codes); $t[16,9]=3, t[32,23]=3$, $t[45,35]=3$. In the present paper: $t[17,10]=3, t[23,15]=$ 3 . Using $\dot{\oplus}$ to denote an amalgamated direct sum (as in
[3]), we also have

$$
\begin{aligned}
& {[27,19] 2 \dot{\oplus}[23,12] 3=[49,30] 5,} \\
& {[42,33] 2 \dot{\oplus}[15,11] 1=[56,43] 3,} \\
& {[42,33] 2 \dot{\oplus}[23,12] 3=[64,44] 5,} \\
& {[49,30] 5 \dot{\oplus}[15,11] 1=[63,40] 6,}
\end{aligned}
$$

which imply $t[49,30]=5, t[56,43]=t[57,44]=3, t[63,40]$ $=6, t[64,44]=5$.

\section{ACKNOWLEDGMENT}

We thank Vera Pless for sending us a preprint of [1].

\section{REFERENCES}

[1] R. A. Brualdi, V. S. Pless, and R. M. Wilson, "Short codes with a given covering radius," preprint.

[2] G. D. Cohen, M. G. Karpovsky, H. F. Mattson, Jr., and J. R. Schatz, "Covering radius-Survey and recent results," IEEE Trans. Inform. Theory, vol. IT-31, pp. 328-343, May 1985.

[3] R. L. Graham and N. J. A. Sloane, "On the covering radius of codes," IEEE Trans. Inform. Theory, vol. IT-31, pp. 385-401, 1985

[4] F. J. MacWilliams and N. J. A. Sloane, The Theory of Error-Correcting Codes. Amsterdam, The Netherlands: North-Holland, 1979.

[5] J. Simonis, "The minimal covering radius $t[15,6]$ of a 6 -dimensional binary linear code of length 15 is equal to 4," IEEE Trans. Inform. Theory, to be published.

[6] T. Verhoeff, "An updated table of minimum-distance bounds for binary linear codes," IEEE Trans. Inform. Theory, vol. IT-33, pp. 665-680, Sept. 1987.

[7] G. J. M. van Wee, "Improved sphere bounds on the covering radius of codes," IEEE Trans. Inform. Theory, to be published. 